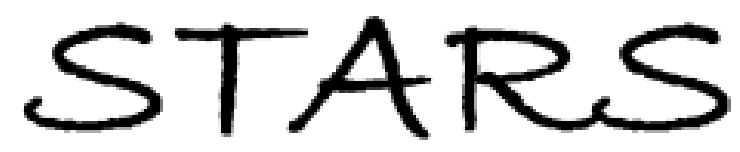

University of Central Florida

STARS

Faculty Bibliography 2000s

Faculty Bibliography

$1-1-2006$

\title{
Glasses for Raman nonlinear optics
}

\author{
G. I. Stegeman \\ University of Central Florida \\ R. Stegeman \\ University of Central Florida \\ C. Rivero \\ University of Central Florida \\ K. Richardson \\ University of Central Florida \\ T. Cardinal
}

See next page for additional authors

Find similar works at: https://stars.library.ucf.edu/facultybib2000

University of Central Florida Libraries http://library.ucf.edu

This Article is brought to you for free and open access by the Faculty Bibliography at STARS. It has been accepted for inclusion in Faculty Bibliography 2000s by an authorized administrator of STARS. For more information, please contactSTARS@ucf.edu.

\section{Recommended Citation}

Stegeman, G. I.; Stegeman, R.; Rivero, C.; Richardson, K.; Cardinal, T.; and Couzi, M., "Glasses for Raman nonlinear optics" (2006). Faculty Bibliography 2000s. 6614.

https://stars.library.ucf.edu/facultybib2000/6614

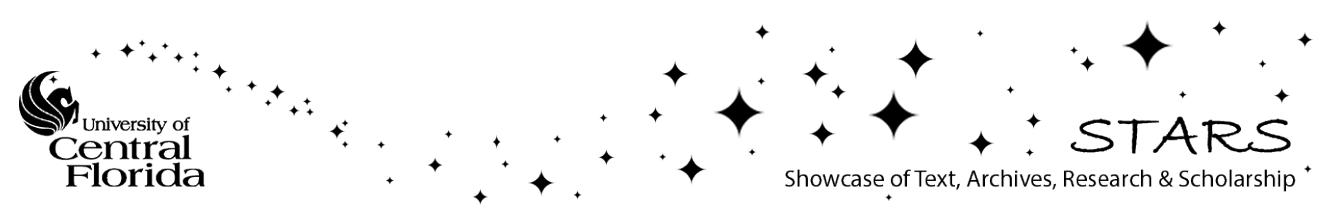




\section{Authors}

G. I. Stegeman, R. Stegeman, C. Rivero, K. Richardson, T. Cardinal, and M. Couzi 


\title{
Glasses for Raman Nonlinear Optics
}

\author{
G. I. Stegeman ${ }^{a, *}$, R. Stegeman ${ }^{a}$, C. Rivero ${ }^{a, b}$, K. Richardson ${ }^{a, d}$, T. Cardinal $^{b}$, and M. Couzi ${ }^{c}$ \\ ${ }^{a}$ College of Optics and Photonics/CREOL and FPCE, University of Central Florida 4000 Central Florida Blvd, \\ Orlando, FL 32816-2700, USA \\ ${ }^{b}$ Institut de Chimie de la Matiere Condensee de Bordeaux, UPR 9048 CNRS 87 Avenue du Dr. Albert Schweitzer, \\ 33608 Pessac cedex, France \\ ${ }^{c}$ Laboratoire de Physico-Chimie Moleculaire, UMR 5803 CNRS University of Bordeaux 1 Bât. A12, \\ 33405 Talence cedex, France \\ ${ }^{d}$ School of Materials Science and Engineering, Clemson University, 161 Sirrine Hall, \\ Box 340971, Clemson, SC 29634-0971, USA \\ *e-mail: george@creol.ucf.edu \\ Received June 16, 2005
}

\begin{abstract}
The Raman effect, by which light is frequency shifted by a vibrational mode, enters into a number of phenomena in nonlinear optics. Here, we summarize our progress in identifying glass materials with potentially useful Raman properties, methods for measuring the strength of the Raman effect and its spectral dependence, and the properties of a number of different families of glasses. Glasses with both larger peak Raman susceptibilities and larger bandwidths relative to fused silica are reported.
\end{abstract}

PACS numbers: 05.45.-a, 42.65-k, 42.65Dr, 52.38Bv, 78.30.Ly

DOI: $10.1134 / \mathrm{S} 1054660 \mathrm{X} 06060028$

\section{INTRODUCTION}

The Raman effect has been used since its discovery in 1928, both to investigate the structure of molecules and to demonstrate phenomena in nonlinear optics [1]. When light interacts with a vibrational mode, optical phonons are excited and light is emitted down-shifted in frequency from the incident light by an amount equal to the vibrational frequency. In the area of nonlinear optics, various phenomena based on this effect have been demonstrated. For example, Bowden and colleagues pioneered the understanding of temporal soliton formation in stimulated Raman scattering [2]. Other effects include Raman gain and Raman lasers, the soliton self-frequency shift, and nonlinear Raman spectroscopies such as RIKES, coherent Raman scattering, etc. $[3,4]$.

Out of all of these different effects, Raman gain used for amplifying a communications signal appears to be the most promising application $[5,6]$. A weak signal can be amplified by a strong pump beam whose frequency is up-shifted from the signal by the Raman response of a material. A key feature of this interaction is that the exponent of the gain depends on the product of the material's Raman gain coefficient and the intensity of the pump beam, so that there is no dependence on the relative phase between the signal and pump [6]. The ideal medium would have (1) a spectrally broad and uniform Raman response which is determined primarily by the Raman susceptibility coefficient $\partial \alpha /\left.\partial Q_{0}\right|_{Q=0}$, where $Q_{0}$ is the vibrational amplitude and $\alpha$ is the molecular polarizability, and (2) a large value for this coefficient. Usefully broad spectral bandwidths are only obtained in disordered materials such as glasses.

The material currently used for Raman amplification in optical systems is based on silica glass, which suffers from both limited bandwidth and relatively small gain, i.e., small $\partial \alpha /\left.\partial Q_{0}\right|_{Q=0}[6,7]$. This has led to progressively more investigations into new glass materials [8-19]. One of the requirements for local area networks is broad bandwidth, high-gain materials in which low loss is desirable but not a critical issue, the socalled Raman in a box [20]. There is also the historic requirement of materials for "long-haul communications" where a combination of low loss, high gain, and large bandwidth is desirable [12]. To date, silica has been the material of choice, only because of its favorable tradeoff between gain and loss.

The search for better materials has been ongoing since the pioneering work of Lines and coworkers in the 1980s [8,9]. However, it is the insatiable appetite of the internet for more bandwidth that has recently accelerated the demand for better materials. A surprisingly large number of glass families have been proposed, and some have been probed experimentally [8-19]. The two standard characterization methods have been spontaneous Raman scattering, in which thermally excited phonons are involved in the process, and direct measurement of Raman gain in fibers [21, 22]. In principle, it is possible to obtain absolute values of $\partial \alpha /\left.\partial Q_{0}\right|_{Q=0}$ from Raman scattering experiments, but it is very difficult to fix and/or measure all of the factors involved. Instead, the Raman spectra are compared to the Raman spectra obtained from fused silica measured at the same 
wavelength. The values for fused silica are reasonably well known [7, 23]. Hence, this process yields the Raman susceptibility of the new material being investigated at that wavelength. Well-known wavelength corrections are then applied to estimate the Raman gain in the communications bands. Obviously, the best approach has been to measure gain directly in fibers at communications wavelengths using various techniques [22]. However, this approach does not lend itself to rapid characterization of materials, since the issue of fabricating low-loss fibers must be solved first.

Recently, we developed an apparatus for making absolute Raman gain measurements for a pump at $1064 \mathrm{~nm}$ [24]. Unlike the spontaneous Raman scattering technique, all of the beam parameters are easy to define, and in fact this apparatus reproduced the value for silica glass accurately as an independent check on its accuracy. Using this apparatus and a relatively standard spontaneous Raman scattering experiment equipped with different excitation (pump) wavelengths, we investigated a number of different glass families with multiple glass compositions in each family. Here, we describe our experiments and the results obtained.

\section{BASIC CONCEPTS}

The Raman effect can occur when the polarizability of a molecule is modulated by one of the vibrational phonon modes of a material. In the molecular frame of reference of a glass made up of " $k$ " distinct units,

$$
\begin{gathered}
\alpha_{i j}^{k}=\sum_{r} \alpha_{i j}^{k, r}\left(\omega_{p}-\omega_{k, r}\right) \\
+\sum_{r} \sum_{\beta}\left\{\left.\frac{\partial \alpha_{i j}^{k, r}\left(\omega_{p}-\omega_{k, r}\right)}{\partial Q_{\beta}^{k}}\right|_{Q_{\beta}^{k}=0}\right\} q_{\beta}^{k},
\end{gathered}
$$

where $\partial \alpha_{i j}^{k, r}\left(\omega_{p}-\omega_{k, r}\right) / \partial Q_{\beta}^{k}$ is the Raman molecular susceptibility, $\omega_{p}$ is the frequency of the incident light, and " $r$ " identifies the different electronic transitions within species " $k$ " which are located at the frequencies $\omega_{k, r}$. Thus, the argument $\left(\omega_{p}-\omega_{k, r}\right)$ describes the frequency dependence of the different contributions to the polarizability, including the Raman susceptibility. The vibrational modes of the $k$ th species are identified by " $\beta$ " and have a frequency $\Omega_{\beta}^{k}$, so that

$$
q_{\beta}^{k}=\frac{1}{2} Q_{\beta}^{k} \exp \left[i \Omega_{\beta}^{k} t\right]+\text { c.c. }
$$

Due to the disordered nature of a glass, for each mode there is a distribution of vibrational frequencies $f_{\beta}^{k}\left(\Omega_{\beta}^{k}-\right.$ $\Omega_{\beta 0}^{k}$ ) which peaks at $\Omega_{\beta 0}^{k}$ with a total species number density $N^{k}$, so that the number density in a frequency interval $d \Omega_{\beta}^{k}$ is given by

$$
N^{k} f\left(\Omega_{\beta}^{k}-\Omega_{\beta 0}^{k}\right) d \Omega_{\beta}^{k},
$$

with the normalization

$$
\int_{-\infty}^{\infty} f\left(\Omega_{\beta}^{k}-\Omega_{\beta 0}^{k}\right) d \Omega_{\beta}^{k}=1 .
$$

Note that here we assume that the spectral breadth of the distribution function is much larger than the natural linewidth due to the decay of the individual modes.

The frequency dispersion in the refractive index reflects the behavior of the linear susceptibility averaged over all of the species present in the glass, their orientations (denoted by \langle\rangle ) and their electronic transitions. From Eq. (1), the refractive index of the material is given by

$$
n^{2}=1+\frac{1}{\varepsilon_{0}} \sum_{k} N^{k}\left(\Re \text { eal }\left\{\sum_{r} \alpha_{i j}^{k, r}\left(\omega_{p}-\omega_{k, r}\right)\right\}\right) \text {. }
$$

(The absorption spectrum is given by the imaginary component.) Hence, the wavelength dispersion in the refractive index is a summation of the dispersion due to all of the electronic transitions in all the component species.

The frequency dispersion of the Raman susceptibility $\partial \alpha_{i j}^{k, r}\left(\omega_{p}-\omega_{k, r}\right) / \partial Q_{\beta}^{k}$ associated with species $(k)$ and a specific Raman line $(\beta)$ in a multicomponent glass clearly does not mirror that of the refractive index except under very special circumstances. Such an equivalence could occur for single-component glasses or glasses with a dominant component, which exhibit a single vibrational mode coupled to the single dominant electronic transition responsible for the linear polarizability. In general, every vibrational mode in species $k$ could couple to multiple electronic transitions in that species with the coupling strength depending on the direction of the transition electric dipole moment and the direction of the vibrational displacement. This is in contrast to the frequency dispersion in refractive index, which is an average over all of the electronic transitions in all of the species. However, in fused silica, Stolen has shown that the measured frequency dispersion in the dominant Raman line mirrors that of the refractive index for wavelengths longer that $500 \mathrm{~nm}[6,12]$. In that case, there is a single specie with a dominant electronic transition which couples to the vibration with a shift of $440 \mathrm{~cm}^{-1}(\Delta v=13.2 \mathrm{THz})$, and all the Raman spectra were measured far from the absorption edge, where dispersion and resonant enhancement in the Raman susceptibility is minimal.

The geometries used in probing the Raman response are shown schematically in Fig. 1. In both cases, the incident (pump) field of frequency $\omega_{p}$ and wave vector $k_{p}$ is written as 


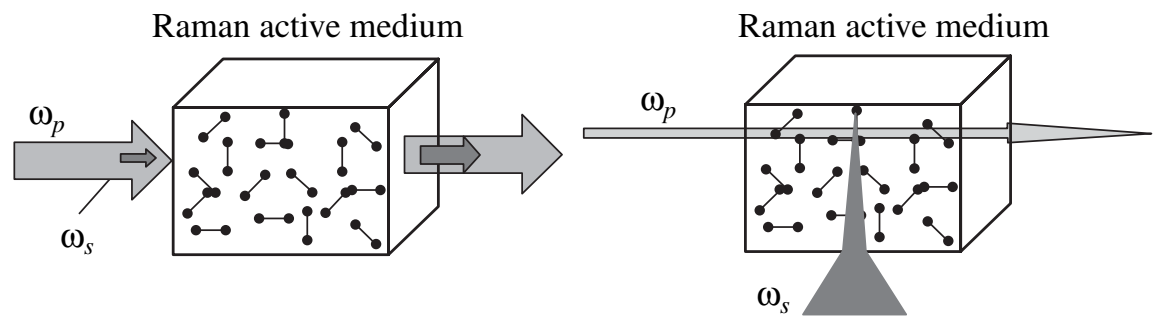

Fig. 1. Schematic representation of the stimulated Raman (gain) process (left) and $90^{\circ}$-geometry spontaneous Raman scattering process (right).

$$
E_{p i}=\frac{1}{2} E_{p i} \exp \left(i \omega_{p} t-k_{p} z\right)+\text { c.c. }
$$

Similarly, the Raman signal (or scattered) field takes the form

$$
E_{s i}=\frac{1}{2} E_{s i} \exp \left(i \omega_{s} t-\mathbf{k}_{s} \cdot \mathbf{r}\right)+\text { c.c. }
$$

where, in this case, $\omega_{s}$ is the signal (scattered light) frequency and $k_{s}$ is the signal (or scattered) wave vector, which lies along the $z$ axis for Raman gain and, for example, for $90^{\circ}$ scattering lies along the $x$ axis in spontaneous Raman. In the Raman gain case, the gain occurs in the overlap region between the copropagating beams, whereas, in the Raman scattering case, light is scattered into all directions, and the scattering volume is defined by the light-gathering optics.

However, there are fundamental differences between the physics of the Raman gain and the spontaneous Raman scattering processes. In Raman gain, the optical phonons are coherently driven by the mixing of optical fields, whereas, in spontaneous Raman scattering, the phonons arise from noise and are hence uncorrelated. For Raman gain, $q_{\beta}^{k} \propto E_{p i} E_{s i}$ with $Q_{\beta}^{k} \propto$ $E_{p i} E_{s i}^{*}[25]$. As a result, the phonons are driven at the frequency $\omega_{p}-\omega_{s}=\Omega \cong \Omega_{\beta}$ (Stokes transition). On the other hand, in spontaneous scattering, the phonon modes are excited by thermal noise and are uncorrelated from molecule to molecule and between different vibrational modes in the same molecule [25]. In this case, due to the statistical nature of the mode excitation, in the high-temperature limit $\left(k_{B} T \gg \hbar \Omega_{\beta}^{k}\right)$,

$$
\left\langle Q_{\beta}^{k} Q_{\beta}^{k *}\right\rangle=\frac{k_{B} T}{m_{\beta}^{k}\left[\Omega_{\beta}^{k}\right]^{2}} .
$$

For the anti-Stokes case, a phonon is annihilated and the frequency is shifted by $\omega_{p}-\omega_{s}=-\Omega \cong-\Omega_{\beta}^{k}$. Here, $m_{\beta}^{k}$ is the effective mass for the vibration. Details can be found in [25].

From this point on, the derivations follow the classical routes described in [25]. In the limit of broad distri- butions in $\Omega_{\beta}^{k}$, i.e., dominated by disorder rather than phonon lifetime, the spontaneous Raman spectrum is given by

$$
\begin{gathered}
\frac{I\left(\omega_{s}\right)}{\Delta \Omega I\left(\omega_{p}\right)}=\sum_{k} N^{k} \sum_{r} \sum_{\beta 0} \frac{\omega_{s}^{4}}{8 c^{4}(4 \pi)^{2} \varepsilon_{0}^{2}} \\
\times\left.\left|\frac{\partial \alpha_{i j}^{k, r}\left(\omega_{p}-\omega_{k, r}\right)}{\partial Q_{\beta 0}^{k}}\right|_{Q_{\beta 0}^{k}=0}\right|^{2} \frac{k_{B} T}{m_{\beta}^{k} \Omega^{2}} f_{\beta 0}^{k}\left(\Omega-\Omega_{\beta 0}^{k}\right),
\end{gathered}
$$

where $\Delta \Omega$ is the solid angle subtended by the detector and the optical parameters are values inside the material. The Raman gain coefficient for the intensity in the plane wave limit, i.e., $I\left(\omega_{s}, z\right)=I\left(\omega_{s}, 0\right) \exp \left[\gamma_{R}(\Omega) I_{p}\left(\omega_{p}\right.\right.$, $z) z$ ] is given by

$$
\begin{gathered}
\gamma_{R}\left(\omega_{p}-\Omega\right)=\sum_{k} N^{k} \sum_{r} \sum_{\beta 0} \frac{\omega_{s} \pi}{2 m_{\beta 0}^{k} n_{s} n_{p} c^{2} \varepsilon_{0}^{2}} \\
\times\left.\left|\frac{\partial \alpha_{i j}^{k, r}\left(\omega_{p}-\omega_{k, r}\right)}{\partial Q_{\beta 0}^{k}}\right|_{Q_{\beta 0}^{k}=0}\right|^{2} \frac{1}{2 \Omega} f_{\beta 0}^{k}\left(\Omega-\Omega_{\beta 0}^{k}\right),
\end{gathered}
$$

where $\Omega=\omega_{p}-\omega_{s}$. Note that the frequency spectra are essentially identical in the limits assumed, except for small differences in the tails. The distributions usually take the form of Voigt functions, which are characterized in the tails of the distribution by Gaussian-like behavior. Hence, the distributions do not "blow up" at low frequencies, despite the $1 / \Omega$ and $1 / \Omega^{2}$ dependence evident in Eqs. (9) and (10) at small frequency shifts. Finally, we note that there is an explicit dependence of these spectra on the pump frequency via $\omega_{s}^{4}$ and $\omega_{s}$ for spontaneous Raman scattering and Raman gain, and implicitly on $\partial \alpha_{i j}^{k, r}\left(\omega_{p}-\omega_{k, r}\right) / \partial Q_{\beta}^{k}$ in both cases, and on $n_{s}$ and $n_{p}$ for Raman gain.

\section{EXPERIMENTAL DETAILS}

\subsection{Samples}

The samples were prepared by conventional melting methods. In the case of the oxide compositions, the samples were melted in platinum crucibles at temperatures 


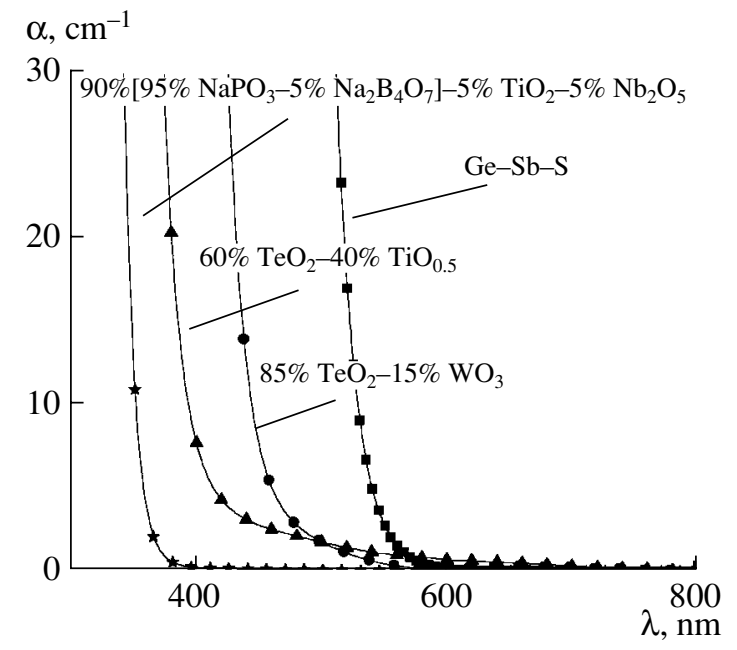

Fig. 2. Example of the relative absorption band edge for a chalcogenide glass (square), $\mathrm{TeO}_{2}$-based glasses (circle and triangle curves represent the extreme band-gap positions for different $\mathrm{TeO}_{2}$ compositions tested to date), and a borophosphate glass matrix (star).

of 900 and $1150^{\circ} \mathrm{C}$ for $30 \mathrm{~min}$ for the tellurite- and borophosphate-based glasses, respectively. In the case of the chalcogenide glass composition, the sample was melted in a rocking furnace in an evacuated, sealed silica tube to avoid any oxygen contamination. After the melt, the samples were annealed at $40^{\circ} \mathrm{C}$ below their glass transition temperature and optically polished.

Figure 2 illustrates the absorption spectra of four different glasses. As one can clearly see from the figure, the absorption band gap of the four different compositions, representative of different glass systems, spans throughout the visible region of the spectrum. In the case of the chalcogenide glass composition, the absorption band edge is in the red, followed by tellurite-based glasses, whose absorption bands go from 400 to $450 \mathrm{~nm}$ (shown in the graph are the upper and lower limits of the absorption band-edge positions representative of all the different tellurite compositions tested to date), and finally the borophosphate-based glass composition, with its absorption band edge in the blue (UV) region.

\subsection{Spontaneous Raman Scattering}

The spontaneous Raman cross-section measurements were conducted using the micro-Raman setup shown in Fig. 3. Two lines from an $\mathrm{Ar}^{+}$laser (458 and $514 \mathrm{~nm}$ ), the 752-nm line from a $\mathrm{Kr}^{+}$laser, and the 1064-nm line from a Nd : YAG laser were used as the excitation sources. The CCD detector used for visible excitation is from ANDOR, model FI UV. This CCD is cooled to $-75^{\circ} \mathrm{C}$ by the Pelletier effect. It is made of 1024 pixels, each one $26 \mu \mathrm{m}$ wide. The CCD used for near-infrared experiments is an InGaAs array from Jobin-Yvon. It is cooled to liquid-nitrogen temperature

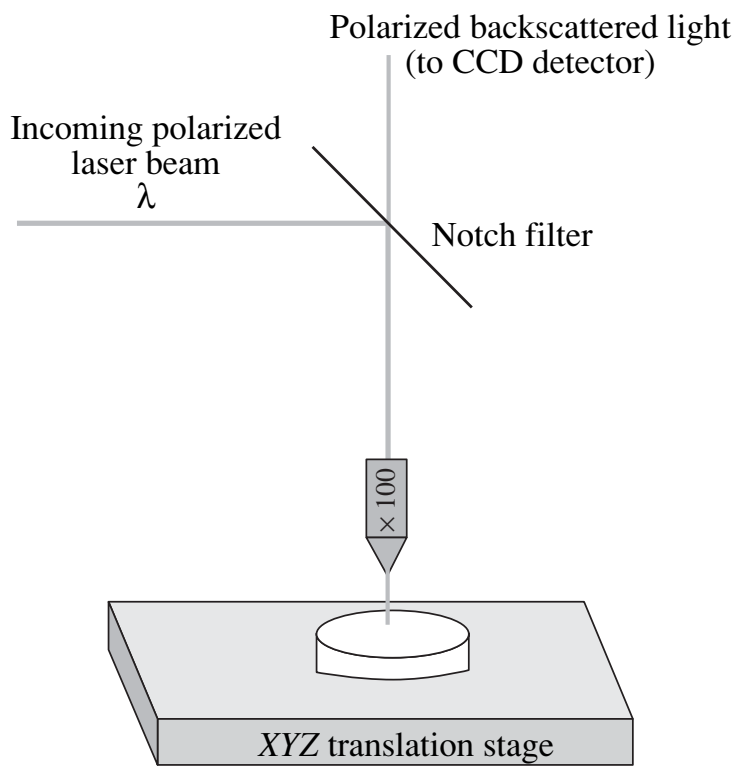

Fig. 3. Geometry of micro-Raman setup. Various lasers and detectors were used at different wavelengths.

and is made of 512 pixels, each $50 \mu \mathrm{m}$ wide. In all cases, the incoming polarized $(\mathrm{V})$ laser beam was focused onto the front polished surface of the sample via a $100 \mathrm{X}$ microscope objective, with a spatial resolution of about $2 \mu \mathrm{m}$. A polarizer was used to select the polarization direction (vertical $\mathrm{V}$ or horizontal $\mathrm{H}$ ) of the scattered light. A backscattering geometry was used to collect the Raman signal, which is spectrally analyzed with a spectrometer and the appropriate CCD detector, with a typical resolution of about $6 \mathrm{~cm}^{-1}$. The Rayleigh line was suppressed with a holographic notch filter.

Furthermore, by measuring the Raman spectra of a test glass under the same experimental conditions as for fused silica at a laser wavelength for which the peak Raman gain for fused silica is known, the peak Raman gain of the test glass at that pump frequency for a Raman active mode can be deduced from

$$
\begin{gathered}
\frac{\gamma_{\beta}^{r, k}\left(\omega_{p}-\Omega_{\beta}^{r}\right)}{\gamma_{\beta^{\prime}}^{r^{\prime}, k^{\prime}}\left(\omega_{p}-\Omega_{\beta^{\prime}}^{r^{\prime}}\right)}=\frac{\left(\omega_{1}-\Omega_{\beta^{\prime}}^{r^{\prime}}\right)^{3}}{\left(\omega_{1}-\Omega_{\beta}^{r}\right)^{3}} \frac{n\left(\omega_{1}-\Omega_{\beta}^{r}\right) n^{\prime}\left(\omega_{1}\right)}{n^{\prime}\left(\omega_{1}-\Omega_{\beta^{\prime}}^{r^{\prime}}\right) n\left(\omega_{1}\right)} \\
\times \frac{\left[1-R^{\prime}\left(\omega_{1}-\Omega_{\beta^{\prime}}^{r^{\prime}}\right)\right]\left[1-R^{\prime}\left(\omega_{1}\right)\right]}{\left[1-R\left(\omega_{1}-\Omega_{\beta}^{r}\right)\right]\left[1-R\left(\omega_{1}\right)\right]} \\
\times \frac{I_{\beta}^{k, r}\left(\omega_{1}-\Omega_{\beta}^{r}\right)}{I_{\mathrm{inc}}\left(\omega_{1}\right)} \frac{I_{\mathrm{inc}}^{\prime}\left(\omega_{1}\right)}{I_{\beta^{\prime}}^{r^{\prime}, k^{\prime}}\left(\omega_{1}-\Omega_{\beta^{\prime}}^{r^{\prime}}\right)},
\end{gathered}
$$

where $R\left(\omega_{p}-\Omega_{\beta}^{r}\right)$ is the Fresnel reflection coefficient [26]. The prime parameters belong to fused silica, for which $\Omega_{\beta^{\prime}}^{r^{\prime}}$ is the peak Raman frequency shift at $440 \mathrm{~cm}^{-1}(\Delta v=13.2 \mathrm{THz})$. 


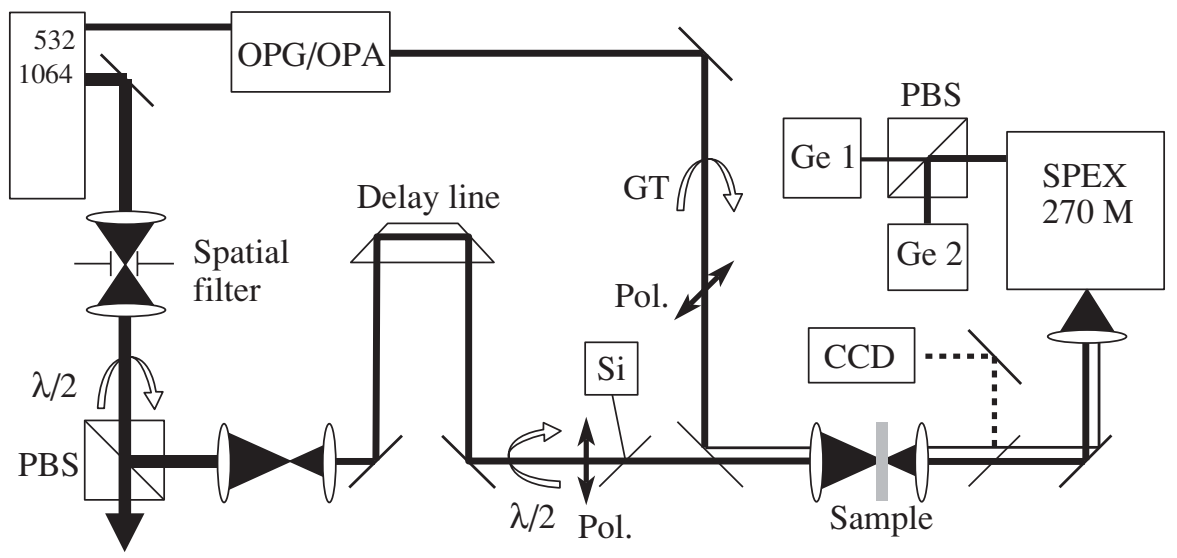

Fig. 4. Apparatus used to measure absolute values of Raman gain.

\subsection{Direct Raman Gain Measurement Apparatus}

The setup used to measure absolute values of the Raman gain coefficient with a 1064-nm pump is shown in Fig. 4 and described in detail in [24].

There are a number of features that allow such absolute measurements using a pulsed laser system consisting of a $10 \mathrm{~Hz} \mathrm{Nd}$ : YAG pump and an OPG/OPA for the signal. Both the Nd : YAG and OPG/OPA pulse widths were measured using autocorrelation techniques, and their temporal overlap inside the sample was optimized. The beams were found to be approximately Gaussian in space and time and were gently focused at the center of the sample (optimized at each wavelength of the OPG/OPA). Thus, a plane-wave approximation was valid over the sample. The OPG/OPA pulse width varied weakly with wavelength (13-16 ps) over the wavelength of interest (1064-1230 nm) and the YAG pulse width was $(33 \pm 2 \mathrm{ps})$. The glass samples, typically $1-$ $2 \mathrm{~mm}$ thick, had their input and output facets polished. Detailed analysis of the spatial and temporal overlap led to the following formula for the measured Raman gain coefficient in the small net signal gain region:

$$
\begin{gathered}
\gamma_{R}(\Omega)=\frac{\Delta E_{T}(\Omega, L / 2)-\Delta E_{S}(\Omega, 0)}{\Delta E_{S}(\Omega, 0) \Delta E_{P}(-L / 2)} \\
\times\left(w_{p}^{2}+w_{s}^{2}\right) \sqrt{\left(\tau_{s}^{2}+\tau_{p}^{2}\right)}\left(\frac{\pi}{2}\right)^{\frac{3}{2}}
\end{gathered}
$$

where $\tau_{p}$ and $\tau_{s}$ and $w_{p}$ and $w_{s}$ are the pump and signal pulse widths and minimum spot sizes, respectively [24]. Knife-edge techniques were used to measure the spot sizes; the corresponding Rayleigh ranges were greater than $10 \times$ the sample size, and the input signal beam spot size was approximately $60 \%$ of the pump beam spot size. Furthermore, $\Delta E_{T}(\Omega, L / 2), \Delta E_{S}(\Omega, 0)$ and $\Delta E_{P}\left(\omega_{P},-L / 2\right)$ are the total pulse energy (Raman + input signal) at the output of the sample, the input signal energy, and the input pump energy, respectively. Surface reflections are obtained from the experimen- tally determined index of refraction data. This is a correction factor which is applied to the measurements along with the depolarization ratio of the material under investigation. Signal pulse energies were measured for input signals both parallel and orthogonal to the pump laser, and depolarization values from the spontaneous Raman experiments were used to estimate $\Delta E_{S}(\Omega, 0)$ from the measured orthogonal output signal. The dispersion with wavelength of all of the optical components, monochromator, etc., was measured and used to correct the Raman gain coefficient. The peak value and wavelength distribution of the Raman gain of silica was measured $\left(0.9 \times 10^{-13} \mathrm{~m} / \mathrm{W}\right)$ with this apparatus and found to agree with the accepted value to within the experimental error.

\section{HIGH-GAIN TELLURITE GLASS SYSTEMS: RESONANT ENHANCEMENT OF THE RAMAN SUSCEPTIBILITY}

Tellurite glasses have been of interest to the glassNLO communities because they have been predicted, and shown, to have large optical nonlinearities [27]. In fact, their Raman gain coefficients have been measured by all three techniques, namely, spontaneous Raman scattering (515-nm pump), with the apparatus discussed above (1064-nm pump), and in fibers (14XX-nm pumps) [13].

Shown in Fig. 5 are the Raman gain spectra obtained for two members of the tellurite family [16]. Note that, depending on the details of the composition, the gain coefficient can be as high as 40-50 times that of the peak of fused silica $[14,16]$. Furthermore, the bandwidth over which significant gain can be obtained is more than twice that of fused silica. Raman gain coefficients measured by spontaneous Raman scattering with a 515-nm excitation (pump) beam have been reported in tellurite glasses that are a factor of two larger than those measured by the direct NLO method described in Subsection 3.3. These were obtained by 
Frequency shift, $\mathrm{THz}$

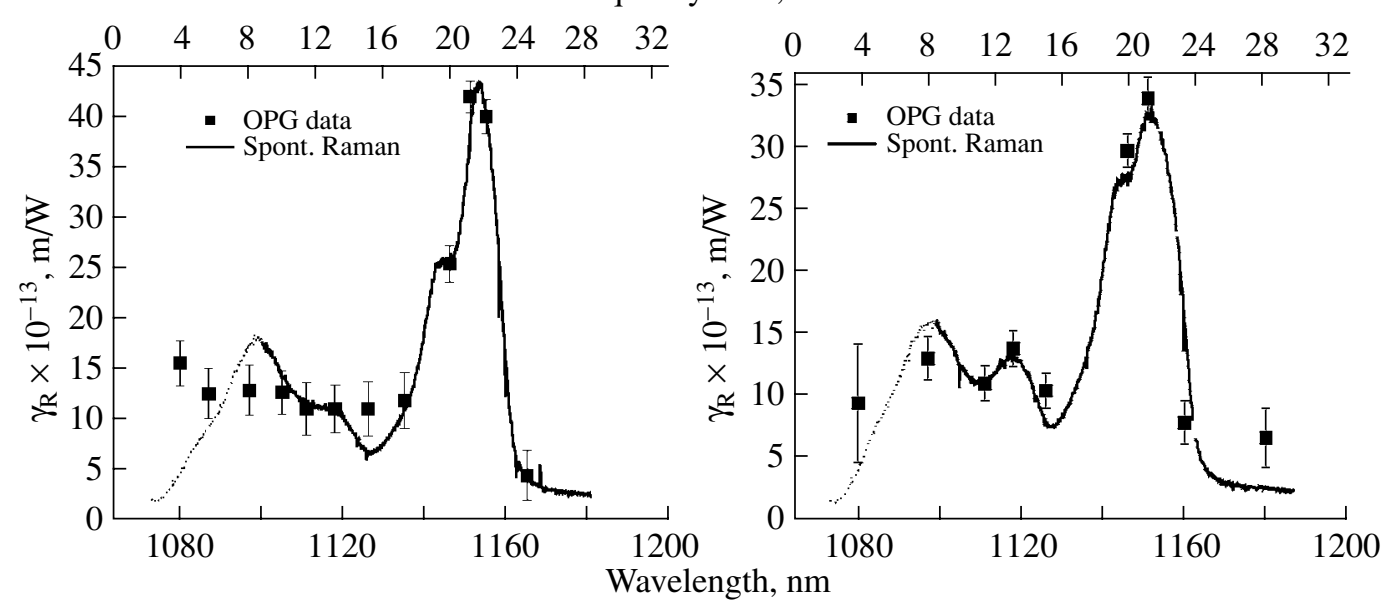

Fig. 5. Raman gain curves of $59.5 \% \mathrm{TeO}_{2}-25.5 \% \mathrm{TlO}_{0.5}-15 \% \mathrm{PbO}$ (right-hand side) and $66.5 \% \mathrm{TeO}_{2}-28.5 \% \mathrm{TlO}_{0.5}-5 \% \mathrm{PbO}(\mathrm{left}-$ hand side). The dashed lines indicate where the notch filter cuts off the spectrum. The spontaneous Raman spectra were normalized to the peak at a $21.3-\mathrm{THz}$ frequency shift.

ratioing their Raman spectra to that of fused silica and using the known value of the silica gain coefficient at $515 \mathrm{~nm}$ to deduce that for the tellurites. The question of these and similar discrepancies has been raised by a number of authors [14, 17-19].

The band edge of two tellurite samples similar to those reported elsewhere is shown in Fig. 2. Given the fact that nonlinear susceptibilities in general are known to disperse with wavelength and that these measurements in the blue-green spectral range are near the band edge, this possibility was investigated by taking the spontaneous Raman spectrum at four different wavelengths, 458, 515, 752, and $1064 \mathrm{~nm}$, and taking the ratio to the fused silica spectrum as described by Eq. (11). In the case of fused silica, its band edge is deep in the UV, and one would not expect significant dispersion in $\partial \alpha /\left.\partial Q_{0}\right|_{Q=0}$ in the visible for fused silica.

The results reproduced in Fig. 6 show a large dispersion in the peak tellurite Raman spectra with pump wavelength between the blue-green visible and $1064 \mathrm{~nm}$. Note, however, that the relative response with increasing pump wavelength becomes wavelengthindependent already in the near-infrared. Because this ratio removes all of the usual wavelength dependence except that found in the refractive index, which is small, and the Raman susceptibility, this dispersion reflects the dispersion in the Raman susceptibility $\partial \alpha /\left.\partial Q_{0}\right|_{Q=0}$. Additional proof in which the shape of the Raman spectrum changes with wavelength in the visible in multicomponent glasses was also reported [26]. Finally, as indicated in Table 1, the values of the Raman gain obtained by the direct NLO measurement technique and that from spontaneous Raman, both measured at 1064-nm pumping, are in excellent agreement. Therefore, this apparent discrepancy has been resolved.
Below in Table 2 is a summary of the directly measured values for the Raman gain coefficient for a variety of glasses in a different tellurite family, which contains lead oxide and thallium oxide. This table illustrates how the peak at $\Delta v=20 \mathrm{THz}$ caused by the vibrations of the $\mathrm{TeO}_{4}$ units and the peak at $\Delta v=21.3 \mathrm{THz}$ caused by the vibrations of the $\mathrm{TeO}_{3}$ and $\mathrm{TeO}_{3+1}$ units vary with molar concentration within the glass matrix. While lead is known to act as a modifier in very small

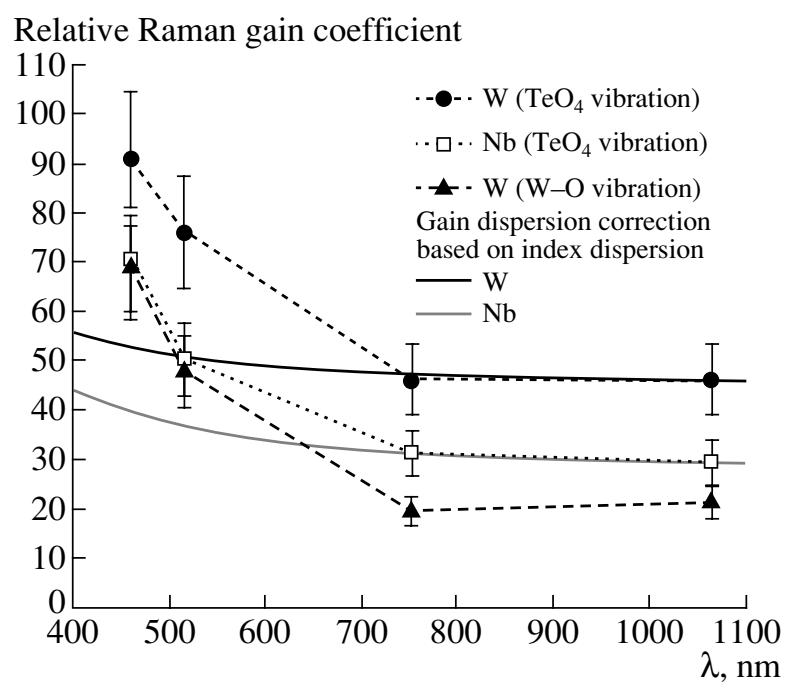

Fig. 6. Estimated multiwavelength Raman gain coefficient at the peak Raman vibration $\left(\mathrm{TeO}_{4}\right.$ units at $665 \mathrm{~cm}^{-1}(\Delta v=$ $20 \mathrm{THz})$ ), and $\mathrm{W}-\mathrm{O}$ vibration (at $920 \mathrm{~cm}^{-1}(\Delta v=$ $27.6 \mathrm{THz}$ )), respectively, normalized to $\mathrm{SiO}_{2}$. The dashed line is used as a guide for the eye. The solid lines represent the $\left(n^{2}(\lambda)-1\right)^{2}$ approximation to the dispersion based on the refractive index dispersion. 
Table 1. Raman gain coefficients with 1064-nm pumping at the peak Raman resonance at $665 \mathrm{~cm}^{-1}(\delta \mathrm{v}=20 \mathrm{THz}) \mathrm{measured}$ by taking the ratio of the spontaneous Raman spectrum to that of fused silica and by direct NLO measurements [26]

\begin{tabular}{l|c|c|c}
\hline \multicolumn{1}{c|}{ Composition, molar percent } & Sample code & $\begin{array}{c}\text { Calculated peak Raman gain } \\
\text { coefficient, from spontaneous } \\
\text { Raman cross-section }\end{array}$ & $\begin{array}{c}\text { Directly measured peak } \\
\text { Raman gain coefficient }\end{array}$ \\
\hline $85 \% \mathrm{TeO}_{2}-15 \% \mathrm{WO}_{3}$ & $\mathrm{~W}$ & $40 \times 10^{-13} \mathrm{~m} / \mathrm{W} \pm 15 \%$ & $38 \times 10^{-13} \mathrm{~m} / \mathrm{W} \pm 10 \%$ \\
$85 \% \mathrm{TeO}_{2}-10 \% \mathrm{Nb}_{2} \mathrm{O}_{5}-5 \% \mathrm{MgO}$ & $\mathrm{Nb}$ & $26 \times 10^{-13} \mathrm{~m} / \mathrm{W} \pm 15 \%$ & $26 \times 10^{-13} \mathrm{~m} / \mathrm{W} \pm 10 \%$ \\
\hline
\end{tabular}

Table 2. Peak Raman gain coefficients of $\mathrm{TeO}_{4}(\Delta v=20 \mathrm{THz})$ units and $\mathrm{TeO}_{3}$ and/or $\mathrm{TeO}_{3+1}$ units $(\Delta v=21.3 \mathrm{THz})[16]$

\begin{tabular}{l|c|c}
\hline \multicolumn{1}{c|}{ Glass composition, Molar percent } & $\begin{array}{c}\Delta v=20 \mathrm{THz} \text { Gain coefficient } \\
\left(10^{-13} \mathrm{~m} / \mathrm{W}\right)\end{array}$ & $\begin{array}{c}\Delta v=21.3 \mathrm{THz} \mathrm{Gain} \text { coefficient } \\
\left(10^{-13} \mathrm{~m} / \mathrm{W}\right)\end{array}$ \\
\hline $66.5 \% \mathrm{TeO}_{2}-28.5 \% \mathrm{TlO}_{0.5}-5 \% \mathrm{PbO}$ & $30 \pm 1.5$ & $34 \pm 1.7$ \\
$63 \% \mathrm{TeO}_{2}-27 \% \mathrm{TlO}_{0.5}-10 \% \mathrm{PbO}$ & $23 \pm 2.6$ & $38 \pm 2.5$ \\
$59.5 \% \mathrm{TeO}_{2}-25.5 \% \mathrm{TlO}_{0.5}-15 \% \mathrm{PbO}$ & $25 \pm 2$ & $42 \pm 1.5$ \\
$75 \% \mathrm{TeO}_{2}-25 \% \mathrm{TlO}_{0.5}$ & $25 \pm 4$ & $19 \pm 3$ \\
$70 \% \mathrm{TeO}_{2}-30 \% \mathrm{TlO}_{0.5}$ & $21 \pm 4$ & $23 \pm 5$ \\
$60 \% \mathrm{TeO}_{2}-40 \% \mathrm{TlO}_{0.5}$ & $21 \pm 5$ & $30 \pm 7$ \\
$50 \% \mathrm{TeO}_{2}-50 \% \mathrm{TlO}_{0.5}$ & $14 \pm 3$ & $52 \pm 3$ \\
\hline
\end{tabular}

molar quantities, it can also serve as an intermediate or partner former in some glass compositions. $\mathrm{PbO}$ is a network participant in these ternary glasses.

\section{CHALCOGENIDES}

Very large values for the Raman gain coefficient (many hundreds of times that of fused silica) have been reported for chalcogenide glass fibers at $1550 \mathrm{~nm}$ [28]. We measured the Raman gain spectrum of a chalco-

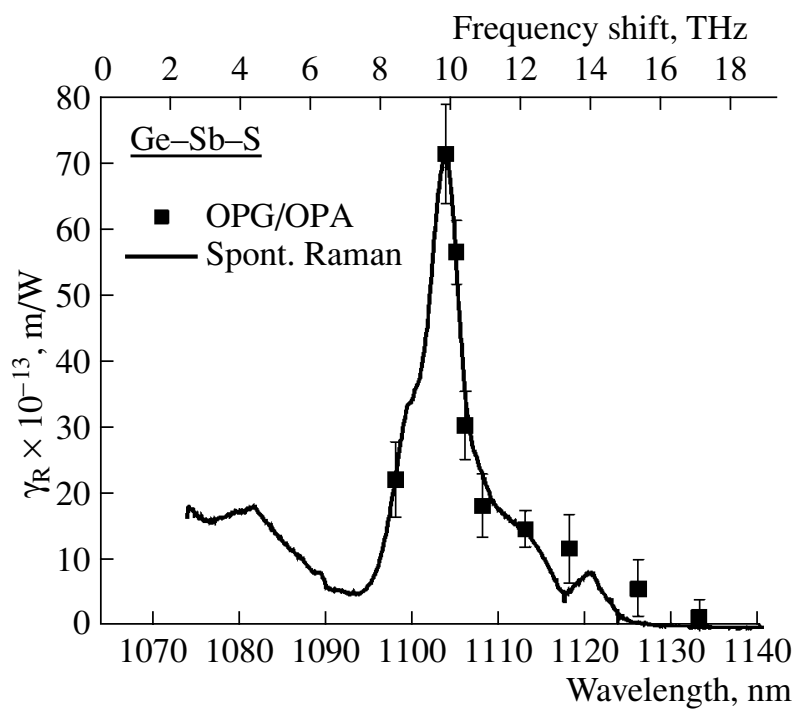

Fig. 7. Raman gain spectrum of the chalcogenide glass Ge$\mathrm{Sb}-\mathrm{S}$ at $1064 \mathrm{~nm}$. genide glass sample in the series $\mathrm{Ge}-\mathrm{Sb}-\mathrm{S}-\mathrm{Se}$ at a 1064-nm pump. The result for a $\mathrm{Ge}-\mathrm{Sb}-\mathrm{S}$ sample is shown in Fig. 7. A peak gain coefficient measured at seventy times that of the peak in fused silica was obtained. The introduction of selenium into the glass matrix red-shifts the absorption band edge; due to this fact, photoinduced structural damage was observed during the experiment. One can account for this issue by using lower pump intensities, since, as has been shown, there is a threshold intensity that is required to activate these light-induced structural changes, previously observed in other selenium-containing glasses [29]. In this case, operation with lower pump intensities is feasible for obtaining reasonable Raman gain signals, since previous spontaneous Raman scattering experiments have indicated that the peak Raman gain was on the order of a hundred times that of fused silica for these compositions. This glass composition had the largest Raman gain coefficient measured in this study.

\section{BROADBAND GLASS: BOROPHOSPHATE-BASED GLASSES}

Experiments were performed on a limited number of glasses in the $90 \%\left[(100-x) \mathrm{NaPO}_{3}-x \mathrm{Na}_{2} \mathrm{~B}_{4} \mathrm{O}_{7}\right]-$ $5 \% \mathrm{TiO}_{2}-5 \% \mathrm{Nb}_{2} \mathrm{O}_{5}$ family, which was designed for broad spectral response. As shown in Fig. 8, although the Raman gain coefficient is comparable to fused silica glass, the gain extends out to almost $40 \mathrm{THz}$ and varies by only about $4 \mathrm{~dB}$ across the full band [30]. This is an increase in bandwidth of more than 5 times that of $\mathrm{SiO}_{2}$. Also, by changing the ratio of the glass constituents, we can tailor the Raman spectrum in order to obtain a more 


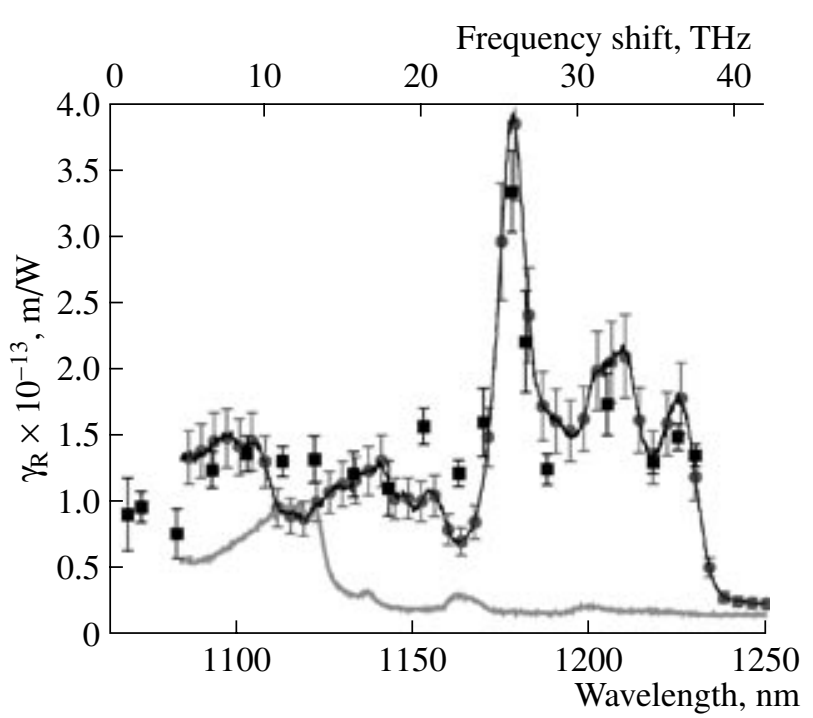

Fig. 8. Measured Raman gain (square) by the direct NLO technique and the normalized spontaneous Raman spectrum for $90 \%\left[95 \% \mathrm{NaPO}_{3}-5 \% \mathrm{Na}_{2} \mathrm{~B}_{4} \mathrm{O}_{7}\right]-5 \% \mathrm{TiO}_{2}-5 \% \mathrm{Nb}_{2} \mathrm{O}_{5}$ (circle). Also shown for comparison is the Raman gain spectrum of fused silica (solid light gray curve).

uniform, flat spectral bandwidth, desirable for this broadband Raman amplification application.

\section{CONCLUSIONS}

Both spontaneous Raman scattering and a new nonlinear optics apparatus capable of absolute Raman Gain measurements at $1064 \mathrm{~nm}$ have been used to measure the Raman gain coefficient in different glasses. One of the key results obtained has been to show that there is dispersion with wavelength in the Raman susceptibility when the measurement wavelength is near the band edge for the corresponding absorption spectrum. As a consequence, measurements of the Raman gain are resonantly enhanced in this case. When the Raman scattering measurements are far from the band edge, the agreement with the direct nonlinear optical measurements is excellent.

The Raman gain properties of a number of glass families have been investigated. It was found that tellurite glasses with different compositions of additional constituents have a Raman gain about 30-50 times that of fused silica, as well as superior bandwidth. The largest gains $>70$ were measured in chalcogenide glasses, although there are issues with photoinduced damage. Borophosphate glasses were found to have gains marginally larger than those of fused silica but with far superior spectral bandwidths, up to 5 times that of fused silica. A number of other glass families are currently being investigated.

\section{ACKNOWLEDGMENTS}

This work was carried out with the support of numerous research, equipment, and educational grants, including NSF grants ECS-0123484 and ECS-0225930 and NSF Integrative Graduate Education and Research Training (IGERT) grant DGE-0114418. The authors also acknowledge the assistance and financial support of the College of Optics and Photonics and the Student Government Association (SGA) at the University of Central Florida. The authors are also thankful for an equipment donation from JDS Uniphase. Finally, the authors would like to acknowledge Phillipe Thomas and Laeticia Petit for providing us with some glass samples for the Raman gain testing.

\section{REFERENCES}

1. C. V. Raman, Indian J. Phys. 2, 387 (1928).

2. J. C. Englund and C. M. Bowden, Phys. Rev. Lett. 57, 2661 (1986).

3. G. P. Agarwal, Nonlinear Fiber Optics, in the series Quantum Electronics-Principles and Applications, Ed. by P. F. Liao and P. L. Kelley (Academic, New York, 1989), pp. 218-262.

4. M. D. Levenson, Introduction to Nonlinear Laser Spectroscopy (Academic, New York, 1982).

5. M. Ikeda, Opt. Commun. 39, 148 (1981).

6. M. N. Islam, IEEE J. Sel. Top. Quantum Electron. 8, 548 (2002); Raman Amplifiers for Telecommunications 1, Physical Principles (Springer, Berlin, 2004).

7. R. H. Stolen, E. P. Ippen, and A. R. Tynes, Appl. Phys. Lett. 20, 62 (1972); R. H. Stolen and E. P. Ippen, Appl. Phys. Lett. 22, 276 (1972).

8. M. E. Lines, J. Appl. Phys. 62, 4363 (1987).

9. M. E. Lines, J. Non-Cryst. Solids 89, 143 (1987).

10. A. E. Miller, K. Nassau, K. B. Lyons, and M. E. Lines, J. Non-Cryst. Solids. 99, 289 (1988).

11. F. L. Galeener, J. C. Mikkelsen, Jr., R. H. Geils, and W. J. Mosby, Appl. Phys. Lett. 32, 34 (1978).

12. R. H. Stolen, "Issues in Raman Gain Measurements," in Tech. Dig. Symposium on Optical Fiber Measurements, NIST Spec. Publ. 953 (National Institute of Standards and Technology, Gaithersburg, MD, 2000) p. 139.

13. A. Mori, H. Masuda, K. Shikano, et al., Electron. Lett. 37, 1442 (2001).

14. R. Stegeman, L. Jankovic, H. Kim, et al., Opt. Lett. 28, 1126 (2003).

15. C. Rivero, K. Richardson, R. Stegeman, et al., J. NonCryst. Solids. 345-346, 396 (2004).

16. R. Stegeman, C. Rivero, K. Richardson, et al., Opt. Express 13, 1144 (2005).

17. G. Dai, F. Tassone, A. Li Bassi, et al., Photon. Technol. Lett. 16, 1011 (2004).

18. G. S. Murugan, T. Suzuki, and Y. Ohishi, Appl. Phys. Lett. 86, 161109 (2005).

19. V. G. Plotnichenko, V. O. Sokolov, V. V. Koltashev, et al., Opt. Lett. 30, 1156 (2005).

20. R. Schafer and J. Jungjohann, Forum-Raman Amplifiers, Compound Semiconductors 7, 41 (2001). 
21. R. W. Hellwarth, Phys. Rev. 130, 1850 (1963).

22. S. Namiki and Y. Emori, IEEE J. Sel. Top. Quantum Electron. 7, 3 (2001).

23. R. H. Stolen, "Nonlinearity in Fiber Transmission," Proc. IEEE 68, 1232 (1980).

24. R. Stegeman, C. Rivero, G. Stegeman, et al., J. Opt. Soc. Am. B (in press).

25. F. A. Hopf and G. I. Stegeman, Applied Classical Electrodynamics, Vol. 1: Linear Optics and Vol. 2: Nonlinear Optics, Wiley Series in Pure and Applied Optics, Ed. by S. S. Ballard and J. W. Goodman (Wiley, New York, 1985).
26. C. Rivero, R. Stegeman, M. Couzi, et al., Opt. Express 13, 4759 (2005).

27. B. Jeansannetas, S. Blanchandin, P. Thomas, et al., J. Solid State Chem. 146, 329 (1999).

28. R. E. Slusher, G. Lenz, J. Hodelin, et al., J. Opt. Soc. Am. B 21, 1146 (2004).

29. C. Lopez, "Evaluation of Photo-Induced Structural Mechanisms in Chalcogenide Glass Materials," PhD Thesis (University of Central Florida, 2004).

30. C. Rivero, K. Richardson, R. Stegeman, et al., J. Glass Technol. (in press). 